\title{
Increased Concomitant Drug AUC
}

National Cancer Institute

\section{Source}

National Cancer Institute. Increased Concomitant Drug AUC. NCI Thesaurus. Code C54613.

Increased area under the plot of plasma concentration of a concomitant drug against time after its administration. 